\title{
Discurso autobiográfico e identidad sociocultural
}

DALIA RUIZ AVILA

UNIVERSIDAD PEDAGOGICA NACIONAL

ESCUELA NACIONAL DE ANTROPOLOGIA E HISTORIA

RESUMEN. El tipo de discurso, la operación discursiva y el parrón de producción texcual son detetminantes para que los sujetos adopten estrategias acordes con la prácrica discursiva que realizan. En este artículo se analizan quince discursos autobiográficos, referentes y expresión de diferentes dimensiones del fenómeno identitario, que pertenecen a tejedores de sombreros de un pueblo maya del Estado de Campeche; se inscriben en la narración como operación discursiva básica y pertenecen al patrón de producción textual oral. Narración y oradidad marcan el funcionamiento del tejido intertextual de este tipo discursivo y destacan símbolos, lugares comunes, espacios, tiempos específicos, auto representaciones (valores y estereotipos), implícitos y figuras retóricas que dan cuenta de las pocencialidades, necesidades y expectativas de los sujetos que interactúan en un ámbito sociocultural determinado. En esta perspectiva teórico-metodológica se inspira la realización de este trabajo; se trata de hacer un análisis del discurso aurobiográfico que permita describir y explicar los mecanismos de consritución y de confirmación de la identidad sociocultural.

PALABRAS CLAVE: discurso autobiografico, auto representaciones, narraciones, identidad sociocultural.

RESUMO. O tipo do discurso, a operacao discursiva e é parrao da produccao rexrual sao fundamentales para que os sujecos adopren estrarexias acordes com a practica discursiva que realizan. Nesse artigo se analizan 15 discursos aurobiográficos, referentes e expresao do diferentes dimensaos da fenómeno identitario que pertenecen a rexedores du sombreiros de um povo da Estado da Campeche, se inscreven na narracao como operacao discursiva básica e pertenecen al patrao da produccao rextual oral. Narracao e oralidad marcan e funcionamento do texido intertextual da este ripo discursivo e destacam simbolos, lugares comunes, espacos, cempos especificos auto representacaos (valores e estereotipos), implíciros e figuras retoricas que dan conta do as porencialidades necessidades e expectativas da os sujetos que interactuan num ámbiro sociocultural determinado. Nessa perspectiva ceórico - metodológica se inspira a realizacao do este trabhajo, se trata do fazer um análisis da discurso aurobiográfico que permita descrever e explicar os mecanismos da constitucao e da confirmacao da a identidad sociocultural.

PALABRAS CHAVE: discurso autobiografico, auto representacaos, narracao, identidad sociocultural. 
ABSTRACT. The type of discourse, the discourse operation and the rextual pattern are fundamental for the subjects to adopt strategies that fit the discourse practice in which they are involved. In this articte are analyzed 15 autobiogtaphical discourses, referents and expression of the various dimensions of the identicy phenomenon; these narrarives by hat weavers from a Mayan town in Campeche, Mexico, are inscribed in narration as the basic discourse process, and in the oral textual production pattern. Narration and orality characterize the intertextual network of this type of discourse. Symbols, common places, spaces, specific moments, selfrepresentations (values and stereorypes), the implicit and rerhorical figures account for the potentialiries, needs and expectarions of the subjects that interplay in a determined socio-cultural environment. This is the theoretical and methodological framework that inspires this paper whose aim is to analize autobiographical discourse in order to describe and explain the mechanisms that lead to the construction and confirmation of sociocultural identity.

KEY WOROS: Autobiographical speech, self-representation, narration, sociocultural identity

"Un día escribí que rodo es autobiografía, que la vida de cada uno de nosotros la estamos contando en rodo cuanto hacemos y decimos, en los gestos, en la manera como nos sentamos, como miramos, como volvemos la cabeza o cogemos un objero del suelo..."

Jose Saramago (1998).

\section{Introducción}

Este artículo se basa en la revisión de quince discursos autobiográficos pertenecientes a hombres y mujeres de Becal ${ }^{l}$. El objetivo que lo inspira es hacer un análisis del discurso autobiográfico que permita el reconocimiento y la descripción del funcionamiento de algunos mecanismos lingüíscico-discursivos que dan cuenta de la constitución y confirmación de la identidad sociocultural presence en esos discursos. Para mostrat algunos aspectos de la realización del fenómeno identitario en el discurso autobiográfico se acude al marco interdisciplinario del análisis de discurso y se constituye un andamiaje teórico metodológico con disciplinas pertenecientes a las ciencias del lenguaje y a las sociales.

Papel relevante juegan: la categoría de operación discursiva y su relación con los patrones de producción textual; las estrategias de estos discursos y sus vínculos con la exposición de núcleos temáticos, que sintetizan los centros de interés en corno a los cuales giran las necesidades y expectativas de los sujecos autobiografiados. Estas interrelaciones se pueden resumir en la siguiente representación. 


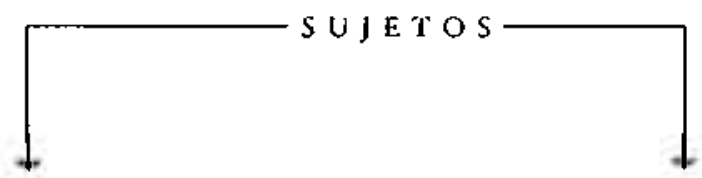

DISCURSO AUTOBIOGRAFICO . . . IDENTIDAD SOCIOCULTURAL

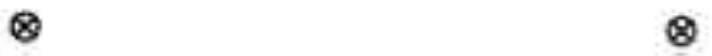

OP. DISCURSIVA. NARRACION

(2)

P. DE PROD. TEXTUAL. ORAL

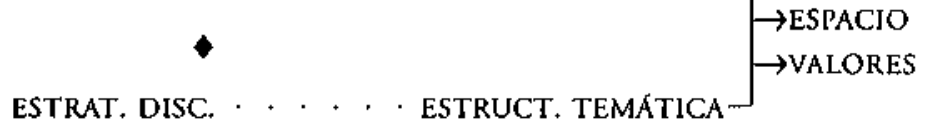

Como puede observarse, el despliegue de este análisis involucra diferentes conceptos; las relaciones que se establecen entre ellos dan lugar a la formación de los siguientes puntos constitutivos de este artículo: 1. Discurso autobiográfico e identidad sociocultural; 2. Operación discursiva y Patrón de producción texcual; y 3 . La estructura temática, una estrategia discursiva.

\section{Discurso autobiográfico e identidad sociocultural}

Algunos referentes del discurso autobiográfico, que son a la vez expresión de diferentes dimensiones del fenómeno idencicario y que en el tejido intertextual suelen manifestarse como: a) símbolos, b) lugares comunes, c) espacios significativos, d) tiempos específicos, e) memoria compartida, f) auto representaciones (valores y estereotipos), g) implícitos, h) figuras recóricas, j) funcionamiento axiológico etc., dan cuenta de las potencialidades, necesidades y expectativas de los sujetos que interactúan en un ámbito sociocultural determinado.

Abordar esca problemática impone la construcción de una definición operativa de discurso en general y de discurso autobiográfico inmerso en una propuesta tipológica, de sujeto y de identidad sociocultural:

1) El discurso es un conjunto transoracional en el que funcionan reglas sintácticas, semánticas y pragmáticas; al igual que de cohesión y de coherencia; también una práctica social peculiar, cuyo funcionamiento constituido por la dinámica de marerialidades diferentes se explica 
por las condiciones de producción, circulación y recepción (Haidar, 2000).

2) En el campo del análisis de discurso es conocida la existencia de diferentes tipos y subtipos de discurso. Esta clasificación suele realizarse a través de un conjunto de criterios, entre los que destacan: objeto discursivo, funciones discursivas, aparatos ideológicos, sujetos de discurso, operaciones discursivas, patrones de producción textual y rasgos de informalidad y de formalidad. Una evaluación a partir de estos elementos teórico-metodológicos permite considerar al discurso autobiográfico como un tipo del cual pueden desprenderse una serie de subtipos como la confesión, el diario, las memorias, la correspondencia epistolar, la historia de vida, etc.

3) El sujeto es un ejecutor de prácticas discursivas ${ }^{2}$, que tiene carácter individual, colectivo, sociocultural, psicológico, etc; que establece relaciones, representa lugares sociales y cuya producción de discursos se da en el marco de formaciones ideológicas, a partir de las cuales se originan las matrices de sentido (Haidar, 1977: 154).

El discurso autobiográfico resulta de la reflexión de un sujeto que en determinadas condiciones de producción discursiva construye, proyecta y sostiene una imagen particular de sí mismo y de los otros, tanto con relación al ser como al estar y al hacer. Su motivación inicial es una reconstrucción de la propia vida y es un tipo de producción discursiva dirigida a un público peculiar (profesor, confesor, psicólogo, investigador social, etc.), su narración generalmente se inscribe en el marco de una inscicución; sin embargo, sus condiciones de producción y de recepción no se agotan ahí, también pueden involucrar en diferences situaciones comunicativas a sujetos con los cuales se tienen relaciones de afectividad y confianza.

El enunciador de este discurso no tiene como objetivo que el otro haga algo dererminado, sino sólo hacerle saber, darle a conocer, quién es él; qué es lo que hace; de qué manera. La significación de un enunciado se concencra en el juego que se establece entre el emisor y el destinatario, comprometidos uno y otro de manera asimétrica en la constitución de la significación completa.

El contenido del discurso autobiográfico constituye una selección de lo que se ha vivido, de la experiencia que sólo se produce dentro de los marcos establecidos por la concepción de pertenencia a la cultura del enunciador. En este sentido, cada autobiografiado es un sujeto creador de un discurso original mediado por las relaciones interdiscursivas $y$ socioculturales.

4) En la concepción de identidad sociocultural juegan un papel determinante los procesos de interacción social, natural simbólica y comuni- 
cativa; a partir de su constitución es posible comprender el lugar de los sujetos en el mundo, referirse al espacio al cual se le confiere un orden, al tiempo que no es sólo lineal sino que se mezcla con lo sagrado, a la naturaleza, al cielo, a la tierra, al agua, a la vegetación y a la vida humana en general. (Ruiz Avila, 1988: xxviii)

En consecuencia, la identidad sociocultural se constituye en un contexto específico de relaciones sociales, es producto de un desarrollo histórico particular que se realiza y manifiesta en dos dimensiones: la personal y la colectiva, y remite a un proceso de inclusión y exclusión; de autodefiniciones y heterodefiniciones que dan cuenta de los sistemas de comunicación, del conjunto de conocimientos y de la visión del mundo que comparten los sujetos pertenecientes a un grupo social y que como corolario los hace diferentes a otros sujetos. Obsérvese el esquema 2.

En esta representación gráfica es posible apreciar que la cuestión de la identidad personal, colectiva, social, cultural, étnica, local, regional, estatal, y nacional, vista desde lo micro a lo macro en las dos grandes dimensiones establecidas, atraviesan al sujeto confiriéndole especificidades, pero también muchos puntos de encuentro con los otros.

La lógica del esquema obedece a que en el primer polo se consideran más los aspectos concernientes al carácter interno del sujeto y en el segundo los más externos vinculados a un espacio, a un territorio. Entre estos dos polos existe una estrecha relación establecida por las múltiples idencidades que están en el sujeto y las redes dinámicas y procesuales que se tejen constituyéndolo.

Esquema 2: TAXONOMIA DE LA IDENTIDAD SOCIOCULTURAL

Carácter multidimensional

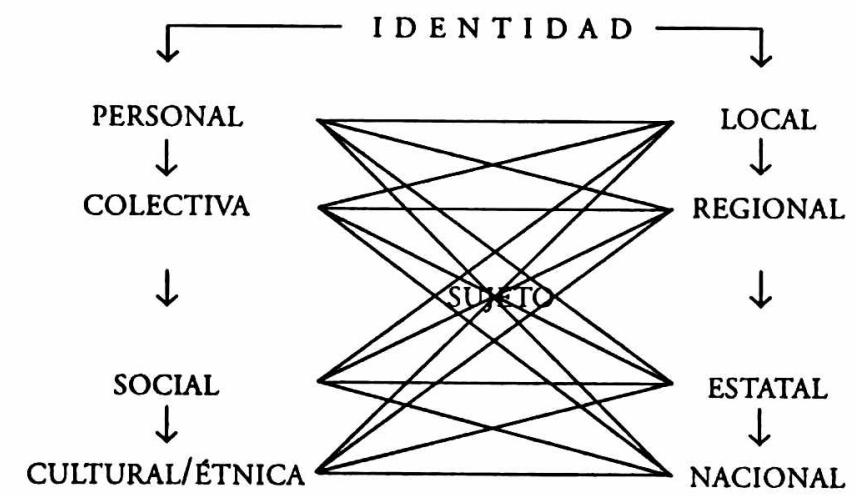

Carácter relacional 
El fenómeno identitario es similar a un caleidoscopio con muchas posibilidades de construcción y de comprensión significativas, por ejemplo "ser becaleño" en muchos contextos es igual a "ser tejedor" porque el primero se identifica con los segundos no sólo por solidaridad, sino también por su esencia "Yo soy de Bécal" territorio en el que adquieren significancia determinados rasgos identitarios (Ruiz Avila, 1998: $\mathrm{xxx}$ ).

\section{Operación discursiva y patrón de producción textual}

La práctica discursiva, entendida en sentido amplio, cuyas posibilidades de producción y recepción se articulan de manera sistemática a las formaciones ideológicas $y$ a las relaciones de poder contenidas en las prácticas discursivas, se materializa a través de una competencia textual inherente al ser humano. Esta competencia la ejercen los sujetos cotidianamente en forma de operaciones discursivas: narración, descripción, argumentación y demostración; para cada una de ellas, el sujeto desarrolla una competencia específica que a su vez puede realizarse en dos patrones de producción textual: la oralidad y la escritura. Por orro lado, esta competencia también implica el conjunto de conocimientos que se poseen, considera las funciones del discurso y las especificidades socioculturales en las que éste se inscribe, es decir, las condiciones sociales de producción y recepción. (Ruiz Avila, 1998: 414). Véase, el siguiente esquema.

Esquema 3: COMPONENTES TEORICO- METODOLOGICOS

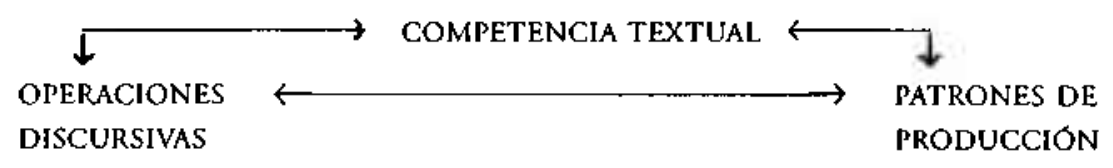

TEXTUAL

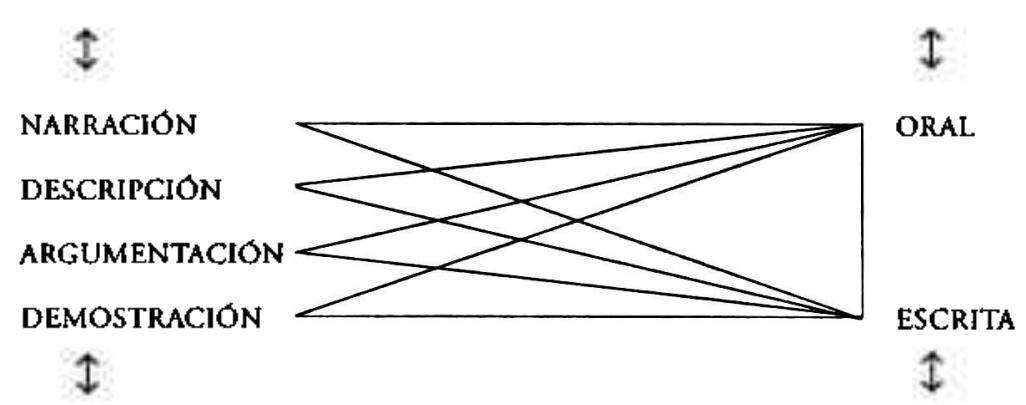

CONDICIONES SOCIALES DE PRODUCCION Y RECEPCION DISCURSIVA 
En el marco de esta propuesta teórica metodológica, una operación discursiva se puede caracterizar de la siguiente manera:

a) Tiene funciones particulares, lo que significa que se relaciona de diferente manera con los sujetos, el tiempo y el espacio del proceso enunciativo.

b) Sostiene vínculos muy estrechos con las otras operaciones discursivas, por ejemplo, no hay una narración que no tenga algún elemento descriptivo, esto es, no hay operación discursiva autónoma, pero sí dominancias acordes al tipo de discurso que se construye, ya sea político, religioso, científico, literario, jurídico, pedagógico, autobiográfico, etc.

c) Presenta una estructura particular, es decir, se sujeta a la presentación de introductores y finales determinados; un desarrollo específico en el que juegan papel particular las microoperaciones discursivas: la justificación y la explicación; reglas de encadenamiento, restricciones, etc.

d) Se realiza a partir del funcionamiento de estrategias discursivas particulares.

En sintesis, una operación discursiva se define vinculada estrechamente a las condiciones de producción y de recepción discursiva y es la realización de un funcionamiento particular del conjunto de elementos lingüístico-discursivos los que permiten distinguir una operación de otra. En ningún discurso hay una operación única, pero si hay una dominante, que se manifiesta a partir del funcionamiento de estrategias propias. La narración es la operación discursiva dominante en el discurso autobiográfico cuyo objeto discursivo es la vida (Ruiz Avila, 1998: 416).

La estructura de esta operación discursiva se configura de la siguiente forma: a) por la relación que se establece en la hiscoria entre los personajes, las acciones, el espacio y el tiempo; b) por el lugar que ocupe el narrador, ya sea en un papel omnisciente o como narrador subjetivo; en el primer caso uciliza la tercera persona, sabe todo lo que dicen, piensan y hacen los personajes; introduce opiniones o comentarios, o se limita a presentar la historia; en el segundo, se apoya en la primera persona y puede ser el procagonista principal del relaco o jugar otro lugar dentro del mismo, pero tiene la misión de telatar lo que ha visto u ofdo desde su punto de vista.

La oralidad, patrón de producción textual de las quince narraciones autobiográficas que constituyen el corpus de esta investigación, en términos generales se caracteriza por propiciar una comunicación espontánea en la que el objero discursivo es la vida; en la realización de este patrón el receptor percibe los elementos lingǘstico - discursivos sucesivamente y tiene la ventaja de la interacción durance la producción del discurso; en este caso destacan las funciones discursivas: emotiva, referencial $y$ poética. 


\section{La estructura temática: Una estrategia discursiva}

El discurso aurobiográfico es resultado de un proceso de selección de las acciones más significativas en donde encran en juego la exclusión, la inclusión desde la memoria, el olvido, el inconsciente, el poder y la ideología; al mismo ciempo, este conjunto de acciones significativas son características, rasgos y criterios que expresan diferencias, marcan contrastes, es decir, sustentan representaciones identitarias.

La conceptualización de estrategia discursiva impone una revisión desde varios ángulos: por ejemplo, la formalización de las operaciones de identificación discursiva, la estructura temática, el funcionamiento de la operación discursiva dominante, los procesos de estereotipización y las formas de realización del implícito en el discurso.

La complejidad del análisis de las estrategias discursivas se relaciona con las diversas realizaciones que éstas pueden adoptar a lo largo de un discurso, por ejemplo: de persuasión; de refutación; de concesión; de atenuación; de confrontación, de orden u organización; de convencimiento; retóricas, en el sentido de funcionamiento de tropos y de figuras de lenguaje; etc. No suelen presentarse en forma pura y transparente y en un mismo tejido discursivo pueden conjugarse diversas estrategias, aunque en términos generales se manifiesta la prioridad de alguna.

El funcionamiento y los mecanismos de las estrategias discursivas se corresponden con el funcionamiento del discurso y en una proyección de lo micro a la macro se vinculan con la situación comunicariva y a las condiciones de producción y recepción del mismo. A partir de este reconocimiento se entiende por estrategia discursiva el conjunto de acciones destinadas a producir cierros efectos de identificación, interpelación, construcción temática y de estructura narrativa de los discursos.

La adopción de una escrategia discursiva pasa en primera instancia por el tamiz de la operación discursiva y en la construcción de un discurso autobiográfico establece una disyunción entre la primera persona y su informe sobre sí mismo. El acto de informar separa el yo que escá contando del yo o los yo pasados que se cuencan, pero también de un yo permanente, "yo he crabajado toda la vida" (13 VITA 62), y un yo parcial "yo sufrí mucho cuando murió mi mamá" (13 VITA 62). El yo que habla - escribe escá, vive en la instancia del discurso en él que procura personificar a un yo creado a partir del recuerdo del pasado (Benveniste, 1976).

En estos discursos, las operaciones de reconocimiento como una de las dimensiones, de la estrategia discursiva pueden referirse al sujeto de la enunciación, al interlocutor o al otro, es decir se correlacionan con los deícricos de persona. Las operaciones que tienen que ver con el sujeto de la enunciación, se manifiestan por un yo personal y por un nosotros 
exclusivo e inclusivo. Entre las operaciones referentes al interlocutor está la interpelación que se realiza generalmente por el pronombre tú o por el reverencial usted, y para las operaciones vinculadas al otro está el pronombre de tercera persona, individual o colectivo.

Orra dimensión a tomar en cuenta en el análisis de las estrategias discursivas es la de la estructura temática, ésta prevalece en el corpus en relación con tres aspectos: tipos de temas cuya exposición es constante en los discursos; tratamiento de los mismos y ausencia de otros temas que podrían parecer relacionados con el discurso (Ruiz Avila, 1998: 427).

Para reconocer los elementos linguístico-discursivos que remiten a la identidad sociocultural, el estudio de la conformación de la estructura temática ha resultado fundamental en el análisis del discurso autobiográfico. En otras palabras, se considera que el establecimiento de núcleos constitutivos de una estructura temática sobresaliente es una estrategia discursiva propia de las narraciones autobiográficas. Feixa (1990), para la realización de un estudio sobre la historia de la juventud en Lleida recoge cien historias de vida, que en su análisis las divide en cuatro grandes apartados: Temáticos, Episódicos, Espaciales y Biográficos (Feixa, 1990: 4). Dentro de este mismo campo de estudio, Signorelli (1995), presenta una comparación sistemática de dos historias de vida e inicia su exploración con el emplazamiento de los temas o bloques temáticos.

De estos dos autores tomamos la idea de localizar los núcleos temáticos, grandes temas presentes en los discursos autobiográficos; que los sujetos abordan de manera profunda y prolongada; que se encuentran muy vinculados con los acontecimientos, y que pueden retomarse a lo largo del tiempo de exposición cuantas veces el aurobiografiado lo considere necesario.

Un discurso autobiográfico puede tener varios núcleos temáricos. De ordinario lo fundamental del tema es posible expresarlo con una palabra. Lo importance es que este sea dominante durante un período del discurso y que las referencias a otros temas se presenten como complementarias. La finalidad de ubicar estos núcleos es ratificar las líneas de convergencias en el discurso autobiográfico y corroborar que la escructura temática de un corpus discursivo implica la consideración de tres aspectos: los tipos de temas, las formas de tratamiento de los temas y la ausencia de algunos de èstos y sus implicaciones.

En el corpus objeto de este análisis, se presentan de manera constante los siguientes núcleos temáticos: 1) trabajo/tejido; 2) familia y relaciones de parentesco; 3) educación formal, estudios; 4) asociaciones (cooperativas de producción y distribución de sombreros, y grupos culcurales); 5) diversiones (ricuales, fiestas colectivas y familiares); 6) migraciones (experiencias propias o de algún miembro de la familia nuclear); 7) lengua (punto de vista sobre cualquiera de las lenguas en contacto); 8) 
espacios y relaciones (la plaza, la calle, etc.) 9) sufrimientos (enfermedades y muerte); y 10) búsqueda de pareja (enamoramiento, noviazgo y relaciones sexuales). Todos ellos forman el tejido del discurso, pero no se agotan en una sola presentación, los sujetos los abordan una y otra vez, cual si fueran fuentes de las que pueden obtener múltiples datos para la construcción de estos discursos.

En el siguiente cuadro se presenta una vista general de los núcleos temáticos abordados por cada informante, lo cual no quiere decir que sean los únicos que existen en estos discursos, por ejemplo, a aparecidos y apariciones se refieren sólo tres informantes, en consecuencia este núcleo temático no se incluye.

Cuadro 1: NÚCLEOS TEMATICOS POR CADA INFORMANTE

\begin{tabular}{|c|c|c|c|c|c|c|c|c|c|c|}
\hline Claves de los informantes 3 & 1 & 2 & 3 & 4 & 5 & 6 & 7 & 8 & 9 & 10 \\
\hline 1.BALA.17 & $\mathbf{x}$ & $\mathrm{x}$ & $\mathbf{x}$ & & $\mathbf{x}$ & $\mathrm{x}$ & $\mathbf{x}$ & & $\mathbf{x}$ & \\
\hline 2.BEBE. 28 & $\mathbf{x}$ & $\mathbf{x}$ & $\mathbf{x}$ & & $\mathbf{x}$ & $\mathbf{x}$ & & & $\mathbf{x}$ & $\mathrm{x}$ \\
\hline 3.SANO.34 & $\mathbf{x}$ & $\mathbf{x}$ & & & $\mathrm{x}$ & & & $\mathrm{x}$ & $\mathbf{x}$ & $x$ \\
\hline 4.REGIO.40 & $\mathbf{x}$ & $\mathbf{x}$ & $\mathbf{x}$ & $\mathbf{x}$ & $\mathbf{x}$ & & & $\mathrm{x}$ & & \\
\hline 5.NELI. 50 & $\mathbf{x}$ & $\mathrm{z}$ & $\mathbf{x}$ & & & & & & & \\
\hline 6.MA.33 & $\mathbf{x}$ & $\mathbf{x}$ & $\mathbf{x}$ & $\mathbf{x}$ & $\mathbf{x}$ & $\mathbf{x}$ & & $\mathbf{x}$ & & $\mathbf{x}$ \\
\hline 7.SAME.34 & $\mathbf{x}$ & $\mathbf{x}$ & & & & $\mathbf{x}$ & & & & $\mathrm{x}$ \\
\hline 8.IRDA.40 & $x$ & $\mathbf{x}$ & $\mathbf{x}$ & $\mathbf{x}$ & & & $\mathbf{x}$ & & & \\
\hline 9.GUDA.44 & $\mathrm{x}$ & $x$ & $x$ & $\mathbf{x}$ & & $\mathbf{x}$ & & & $\mathbf{x}$ & $\mathrm{x}$ \\
\hline $10 . \mathrm{GODO} .65$ & $\mathbf{x}$ & $\mathrm{x}$ & $x$ & $\mathrm{x}$ & & & $\mathbf{x}$ & $\mathbf{x}$ & & \\
\hline 11.ANTI.84 & $\mathbf{x}$ & $\mathbf{x}$ & $x$ & & & & & & & $\mathrm{x}$ \\
\hline 12.VANO.94 & $\mathbf{x}$ & $\mathbf{x}$ & $x$ & $\mathbf{x}$ & & & & $x$ & $\mathbf{x}$ & \\
\hline 13.VITA.62 & $\mathbf{x}$ & $\mathbf{x}$ & $\mathbf{x}$ & & $\mathbf{x}$ & $\mathbf{x}$ & $x$ & $\mathbf{x}$ & $\mathbf{x}$ & \\
\hline 14.PAMA.83 & $\mathbf{x}$ & $\mathrm{x}$ & & & $\mathrm{x}$ & & $\mathbf{x}$ & & $\mathbf{x}$ & $\mathbf{x}$ \\
\hline 15.PEILA.93 & $\mathrm{x}$ & $\mathbf{x}$ & $\mathbf{x}$ & & & & $x$ & & $\mathbf{x}$ & \\
\hline TOTAL & 15 & 15 & 12 & 6 & 7 & 6 & 6 & 6 & 8 & 7 \\
\hline
\end{tabular}

Fuente. Corpus Discursos autobiográficos (Ruiz 1998).
1. Trabajo
3. Estudios
2. Familia y relaciones de parenresco
5. Diversiones
4. Asociaciones
7. Lengua
6. Migraciones
9. Sufrimiento (enfermedades y muerte)
8. Espacios y relaciones
10. Búsqueda de pareja (relaciones sexuales) 
Vale aclarar que no se expone el orden en el que los núcleos temáticos se presentan, pues varía de un discurso a otro, por ejemplo: 1 BALA.17 y 2 BEBE. 28 comienzan su relato con pasajes de su trayectoria como estudiantes; 7 SAME. 34 al principio aborda su trabajo como vendedora ambulante, como tejedora, como ama de casa, etc. y 14 PAMA 83 empieza recordando que le gustaba bailar salir en las comparsas, divertir$\mathrm{se}^{4}$.

Los núcleos temáticos constitutivos de estos discursos, que se encuentran más relacionados con la identidad sociocultural, son el trabajo, la familia y los estudios, los dos primeros presentes en todos los discursos, el tercero (ausente en dos discursos) tiene el segundo lugar en frecuencia; los núcleos cinco y diez tienen el cuarto y así sucesivamente. Mediante su desarrollo se percibe que en la constitución de identidad a) entran en juego razones y motivos internos que se combinan con causas exrernas, $y$ b) se hace referencia a configuraciones colectivas, sociales y culturales (Lisón, 1994: 99)

En términos generales, las referencias al trabajo lo presentan como un acto ejecutado con buena fe, constancia y conciencia de obligación. Los becalenos lo asimilan como una labor cotidiana, dura y lenta que si se realiza con constancia y se refuerza con una actitud espiritual de fe cristiana, les proyecta un horizonte de cambio social. Lo dicho con relación a la familia permite definir a ésta en cuanto a su estructura y función; primero por el conjunco de nexos que cohesionan al grupo de parientes y las reglas que rigen la interacción que se produce entre ellos; segundo como la unidad básica de supervivencia social, pues además de cumplir con la reproducción biológica, propicia la socialización y es un referente social (Nolasco, 1981: 45).

La indianidad es tradicionalmente la expresión de una condición social que se relaciona con la campesinidad y el uso de una lengua. Bécal es un territorio con una población campesina e india, en el que la lengua como elemento culcural estratégico está sujeta a cambios. En la exposición de este núcleo temático se puede observar cómo el español actualmente es reivindicado por integrantes de las tres generaciones. Para estos sujetos continuar como hablantes de maya equivale a mantener un estatus de subordinación y de dependencia, del cual quieren librarse.

Trabajo, familia y lengua son núcleos temáticos en cuyo desarrollo los sujetos plasman que a pesar de la aceptación resignada de su condición social, ésta no es suficiente, por lo tanto cada uno, incluso de manera particular, trate de borrar las marcas identitarias que permiten conocer objetivamente quién es indio y quién no lo es.

Otros núcleos temácicos, también vinculados con la identidad, aunque en un rango menor que los tres anteriores son espacios y relaciones, sufrimiento y búsqueda de pareja. De los primeros los más mencionados 
en estos discursos son la casa, la cueva, la milpa, el patio, el parque, la escuela y la calle, como lugares acostumbrados, frecuentados e incluso adaptados a las necesidades de los sujetos que en ellos interactúan. Por ejemplo, las relaciones que se suscitan en las cuevas entre los integrantes de una familia, los amigos, los enamorados y las visicas son expresiones que marcan las diferencias de lo que subsiste, singulariza y conforma la identidad sociocultural de estos sujetos (Ruiz Avila, 1998: 431).

Mediante la exposición de los núcleos temáticos se engarza estructura social e historia; fundamentos y procesos de conocimientos con experiencia; y se presentan a la distancia las líneas de continuidad en el cambio, aunadas a los procesos de permanencia. En este sentido es posible apuntar que muchos de los rasgos materiales de la cultura maya están en proceso de desaparecer, pero también son bastantes las formas de representación propias de esta cultura que aun permanecen inmutables.

Un agrupamiento de los informantes por generaciones y géneros posibilita 1) destacar lo que dicen en relación con los temas que abordan, 2) reconocer las diferencias de tratamiento entre las diferentes generaciones y los diferentes géneros 5 .

Cuadro 2: NÚCLEOS TEMATICOS Y GENERACIONES

\begin{tabular}{|c|c|c|c|c|c|}
\hline & & & ABORDAN & COINCIDEN & DIFIEREN \\
\hline \multirow[t]{7}{*}{ JOVENES } & MUJERES & 1.BALA. 17 & 7 & & \\
\hline & & 2.BEBE. 28 & 7 & & \\
\hline & & & & 6 & 1 \\
\hline & HOMBRES & 3.SANO 34 & 6 & & \\
\hline & & 4.REGIO. 40 & 6 & & \\
\hline & & 5.NELI. 50 & 3 & & \\
\hline & & & & 2 & $3^{*}$ \\
\hline \multirow[t]{5}{*}{ ADULTOS } & MUJERES & 6.MANA.33 & 8 & & \\
\hline & & 7.SAME.34 & 4 & & \\
\hline & & 8.IRDA.39 & 5 & & \\
\hline & & 9.GUDA.44 & 7 & & \\
\hline & & & & 2 & $4^{*}$ \\
\hline \multirow[t]{8}{*}{ ANCIANOS } & HOMBRES & 10.GODO.65 & 6 & & \\
\hline & & II.ANTI.84 & 4 & & \\
\hline & & 12.VANO.94 & 6 & & \\
\hline & & & & 3 & $3^{*}$ \\
\hline & MUJERES & 13.VITA.62 & 8 & & \\
\hline & & 14.PAMA.83 & 6 & & \\
\hline & & 15.PEILA.93 & 5 & & \\
\hline & & & & 4 & $3^{*}$ \\
\hline
\end{tabular}

Fuence. Corpus. Discursos Autobiográficos (Ruiz 1998)

*: Los cemas son abordados sólo por un informante. 
Obsérvese un ejemplo de la realizaciòn del núcleo remático de la lengua presente en los discursos de las tres mujeres ancianas. Todas reconocen el maya como lengua materna y aprendieron el español cuando ya eran adultas. Al referirse a la cuestión de la lengua son conscientes de la significación que atribuyen a ésta. Por ejemplo, palabras más palabras menos, expresan la siguiente fórmula: la comunicación en lengua maya confiere mayor seguridad y en español menor confianza.

La variante generacional es eficaz para mostrar el desplazamiento de una lengua como consecuencia de un conjunto de ideologías que comparten los hablantes, las cuales no son propiciadas únicamente por el gobierno con sus programas de castellanización, bilingüismo y biculturalismo, sino que se generan también en la vida cotidiana del pueblo y de la familia. Por ejemplo, es un hecho que actualmente parientes, patrones o integrantes de la generación de los jóvenes ejercen mecanismos de menosprecio y persuasion sobre los hablantes de maya, la lengua indigena de la región. Estas acciones son parte de un proceso histórico que ha influido para que el uso de esta lengua se reduzca al ámbito familiar, del mismo modo que a espacios en los que entre los interlocutores existen confianza y lazos de afecto.

En la exposición de este núcleo temático resalta el papel básico de la lengua como identificadora cultural. Bécal es un espacio formado por una red de relaciones sociales que expresa su complejidad de diversas formas, una de éstas es la lingǘrstica, en este contexto, el contacto mayaespañol no es un vinculo aislado, está inserto en el marco de las características estructurales del pueblo y los cambios que en cuanto a su uso se derivan pueden verse como referentes implícitos de las reivindicaciones de su ser indio.

Rendón (1992: 30) apunta "En la medida en que cada grupo usa una lengua, esta lo representa, lo identifica y es uno de sus más importances símbolos de identidad". En consecuencia la lengua es un medio de identificación o de referencia al interior de cada grupo $y$ en relación con otros, al mismo tiempo que es un símbolo de identidad por las implicaciones sociales y económicas vinculadas a su uso, por ejemplo, la atención que en general se les dispensa a los hablantes de lenguas indígenas. De acuerdo con la cita de este autor, es posible apuntar que: actualmente en Bécal 1) se están dando cambios fundamentales en el terreno lingüístico, transición del maya al español, mismos que se manifiestan en las expresiones de los representantes de las cres generaciones, al referirse a ella, y 2) que estos cambios repercuten en la identidad, generando modificaciones en el sentido de ésta.

Para concluir este punto es perrinence subrayar que después de examinar los 15 discursos aurobiográficos parece que todos los sujetos, 
en función de su edad y de su género, tienen reacciones similares y adoptan una posición determinada ante temáticas particulares. Por ejemplo. "Yo soy trabajador, yo no soy flojo" (10.GODO.65) "Este trabajo es bonito, pero del sombrero no se puede vivir" (8.IRDA.39) "Por mis hijos yo hago cualquier sacrificio" (9.GUDA.44) "Que mis hijos tengan su profesión" (13.VITA.62)"Yo sufro mucho cuando me voy a otro lado" (7.SAME.34) "Hay que organizarnos para poder defendernos de los comerciantes" (8.IRDA.39).

Existe relación entre los núcleos temáticos abordados y la construcción de la autoadscripción-autodefinición y de la heteroadscripción y la heterodefinición. De la misma forma se puede considerar la pluralidad o presencia de los diversos roles que cada sujeto juega: la asimetría que se establece entre los roles y la conmutación, como la posibilidad de cambios.

Es evidence que en el discurso aurobiográfico se manifiestan vínculos definidos entre el trabajo que tealizan los sujetos y la identidad. En otras palabras, el trabajo genera la creación de valores que permiten escoger si se sigue siendo lo mismo o se intentan nuevos rumbos. Por ejemplo, la esencia del ser becaleño está fundada en el mito del sombrero, del tejedor.

A partir de lo anterior se apunta que en el análisis de la estructura temática de un corpus discursivo de tipo autobiográfico, se encuentran índices sintomáticos de las condiciones de vida de los sujetos; no sólo la presencia y la ausencia de determinados temas, sino también la forma de tratamiento de los mismos, lo cual está determinado por las condiciones de producción y de recepción discursiva, al igual que por la situación comunicativa.

\section{Conclusiones}

De los aspectos abordados en el desarrollo de este artículo es posible presentar a manera de síntesis los siguiences puntos:

- La imagen de sf mismo es generada desde una situación comunicariva y a la vez constitutiva de la operación discursiva narrativa. La representación de sí mismo, contenida en el discurso autobiográfico, varía según los atributos que el sujeto desee, o se sienta obligado a materializar, de acuerdo a sus posibilidades y a la relación social específica de la que participa y de la cual posee, proyecta o exhibe una serie de características que tienden a sostener y otorgar credibilidad a esa imagen.

- Toda aproximación a una vida particular siempre se refiere a un contenido amplio, pero nunca es suficiente ni completa la recupera- 
ción de acciones y detalles, casi infinitos, que componen una vida. Cuando se cuenta la vida generalmente se hace acopio de fragmentos, de retazos de hechos prefabricados por un enfoque y una perspectiva peculiar, desde los cuales se hacen selecciones, omisiones, encadenamientos, atribuciones, explicaciones de causalidad, etc. que enmarcan a un personaje; esto quiere decir que la construcción de un texto autobiográfico no responde a formas ilimitadas ni azarosas; corresponde a operaciones y estrategias discursivas relativamente aceptadas y compartidas socialmente.

- Los núcleos que conforman la estructura temática del discurso autobiográfico de los tejedores dan cuenta de la dimensión simbólica de las prácticas sociales que se producen y se reproducen en el interior de este grupo social.

- Para analizar un discurso autobiográfico es de primera importancia distinguir el papel y las características de las condiciones materiales y simbólicas de su producción. Ellas consisten en el conjunto de rasgos propios de la situación comunicativa, de la relación de interlocución de la cual nace el discurso autobiográfico.

- El discurso autobiográfico se construye en torno a estrategias y valores que las convierten en verdaderas autorrepresentaciones, aporta información sobre normas, reglas, pautas que generan y regulan la interacción social; valores, juicios sobre atributos percibidos y caminos hacia un objetivo que se asocia con una accitud, tales evaluaciones pueden incluir dimensiones como bondad, honestidad, y su intensidad varía según el juicio de valor y prácricas sociales.

Por último, vale sef́alar que a través de este análisis en el que se muestra la relación encre sujetos y núcleos temáticos también es posible conocer la vida y los sentimientos del pueblo de Bécal; sus condiciones materiales de existencia, su experiencia histórica, sus sistemas de normas y valores, sus expectativas de acción presentes y futuras; en fin un conjunto de rasgos que conducen a conocer una amplia dimensión de su identidad sociocultural.

\section{NOTAS}

1. Pueblo de tejedores de sombreros y artesanlas de jipi $y$ wano que se encuentra en el sudeste mexicano, en el Estado de Campeche, en la región de los Chenes y en el municipio de Calkinf.

2. La práctica discursiva es una prácrica social peculiar que acompaña a las otras prácticas sociales y que al igual que ellas produce y reproduce la vida social en rodas sus dimensiones. 
3. Se tuvo especial cuidado en considerar todos los núcleos que aparecieran cuando menos en seis discursos.

4. En una exposición más amplia, Tejiendo discursos se tejen sombreros. Identidad y prácrica discursiva Ruiz Avila, D. (1998), Tesis doctoral, ENAH, México; se hace un análisis de la forma en la que los sujetos abordan los diferentes núcleos temáticos y se contrasta con las variables de educación formal, género y generación.

5. Esca clave se compone de un número que indica orden; de un seudónimo formado por las dos primeras, más las dos últimas letras del nombre del sujeto autobiografiado y de su edad.

\section{REFERENCIAS BIBLIOGRAFICAS}

Benveniste, E. (1976). Problemas de Lingiüstica General, (6a. ed. en español). México: Ed. Siglo XXI.

Fetxa, C. (1990). Discurso autobiográfico e identidad generacional. La juventud como metáfora. Ponencia presentada en V Congreso de Antropologia, Granada, España.

HaIdAR, J. (1997). Las materialidades discursivas y su funcionamiento: problemas teórico metodológicos. En Bolivar A, y Bentivoglio P. (Eds.). Actas del I Coloquio Latinoamericano de Analistas del Discurso (pp. 153-162). Caracas: Comisión de Estudios de Posrgrado, Universidad Central de Venezuela.

Haidar, J. (2000). Elementos teórico-metodológicos del análisis de discurso. En Ruiz Avila, D. (coord). Serie de TV Discurso y Educación, Programa 1, Sistema de Televisión Educativa. México: EDUSAT, UPN.

LIsón, T. C. (1994.) ldenridad: collage cultural, las múltiples voces de la identidad. En Avila P. R. y Calvo B. T. (Comps.) Identidades, nacionalismos y regiones (pp: 80-105). México: Universidad de Guadalajara y Universidad Complutense de Madrid.

Nolasco, M. (1981). Modelos estructurales de las familias pobres de las ciudades de México. Revista Trabajo Social, 43-58. México: UNAM.

Rendon, J. (1992). Noras sobre idencidad, lengua y cultura. En Méndez L. (Comp.) Seminario sobre identidad (pp. 28-49). México: Instituto de Investigaciones Antropológicas, UNAM.

RuIZ AVILA, D. (1998). Tejiendo discursos se tejen sombreros. Identidad y práctica discursiva, Tesis doctoral. México: Escuela Nacional de Antropología e Hiscoria.

Rulz Avila, D. (2000). Cuentame tu vida. Compendio de discursos autobiográficos. México: Universidad Pedagógica Nacional.

Saramago, J. (1998). Cuadernos de Lanzarote, 1993-1995. México: Edicorial Alfaguara.

Signorellu, A. (1995). El valor del trabajo en la experiencia biográfica, confrontación de dos historias de vida comparadas. Cuicuilco, vol, 2 (4): 123147. México: Escuela Nacional de Antropología e Historia. 
Dalla RUIZ AVILA es docente e investigadora de la Universidad Pedagógica Nacional y profesora de la Escuela Nacional de Antropología e Hiscoria; en la primera institución es Coordinadora de la línea de investigación Formacion docente y prácticas institucionales, del doctorado en Educación. Es Licenciada y Maestra en Lingüistica y Doctora en Antropología. Las líneas de investigación que ha atravesado en su trayectoria académica son Discurso y Educación y Discurso e Identidad sociocultural, ambas en el marco del análisis de discurso y de la semiótica de la cultura. De esta incursión ha desprendido diversos artículos y en el 2001 se publicó su libro Cuéntame tu vida. Compendio de discursos autobiográficos, Universidad Pedagógica Nacional, México.

Correo electrónico: dravila@servidor.unam.mx 